\title{
Ti:sapphire rib channel waveguide fabricated by reactive ion etching of a planar waveguide
}

\author{
A. Crunteanu, G. Jähnchen, R.P. Salathé, P. Hoffmann, and M. Pollnau, \\ Institute of Applied Optics, Department of Microtechnique, \\ Swiss Federal Institute of Technology, CH-1015 Lausanne, Switzerland
}

\author{
R.W. Eason and D.P. Shepherd \\ Optoelectronics Research Centre, University of Southampton, \\ Highfield, Southampton SO17 1BJ, United Kingdom
}

We were successful in creating $1.4-\mu \mathrm{m}$ high ribs in a Ti:sapphire planar waveguide by reactive ion etching. Optical investigations of the obtained structure showed channel-waveguide fluorescence emission of the Ti:sapphire layer after Ar-ion excitation.

Recently, Ti:sapphire planar waveguides were fabricated [1] by pulsed laser deposition on sapphire substrates. Due to their high crystallinity, optical quality, and low-loss propagation laser oscillation was successfully demonstrated [2]. Such planar waveguides can also be used as coherent broadband emitters for interferometric applications [3]. Fabrication of a channel waveguide by structuring of such a planar waveguide would allow us to obtain fluorescence emission with improved coherence and efficiency in the case of broadband emission and a reduced pump threshold and increased output power in the case of laser oscillation.

Here we report, to our knowledge, the first channel-waveguide emission from a Ti:sapphire structure. We engraved channels of $1.4-\mu \mathrm{m}$ depth in a $10-\mu \mathrm{m}$ thick $\mathrm{Ti}(0.1 \%)$ :sapphire planar waveguide by reactive ion etching (RIE). RIE is a well-established method for patterning semiconductor materials, but until recently $[4,5]$ less attention was paid to the fabrication of microstructures in sapphire or Ti:sapphire.

Fabrication of structures with low surface roughness is essential for receiving low-loss propagation in channel waveguides. First, we investigated the suitability of RIE and the structural properties of the etched areas in c-cut sapphire substrates. We spin-coated a polyimide mask of $12-\mu \mathrm{m}$ thickness onto the surface of the sapphire substrate. This mask was structured by laser ablation with an $\mathrm{ArF}^{*}$ excimer laser at $\lambda=193 \mathrm{~nm}$. In this way, we defined channels of $100-\mu \mathrm{m}$ width, $12-\mu \mathrm{m}$ depth, and distanced by $15-\mu \mathrm{m}$ in the polyimide. RIE was then performed in a $\mathrm{BCl}_{3}: \mathrm{Cl}_{2}$ atmosphere at $3 \mathrm{mT}$ Torr pressure by use of an inductive plasma system. The etch rate of the sapphire was $45 \mathrm{~nm} / \mathrm{min}$ and the etching selectivity between the sapphire and the mask was 1:3.5. The obtained structures were investigated by profilometry. Additional results obtained by atomic force microscopy (AFM) show that the roughness of the sapphire substrates is lowered from an rms value of $15.9 \mathrm{~nm}$ to an $\mathrm{rms}$ value of $3.7 \mathrm{~nm}$ for the etched regions.

The same experimental conditions were used for the RIE of the Ti:sapphire planar waveguide. Figure 1 shows the rib profile of one of the RIE-etched regions of the Ti:sapphire planar waveguide. 
We investigated the Ti:sapphire rib structures by optically pumping the waveguide with an Ar-ion laser that operated $\mathrm{cw}$ on all lines. The pump light was focused into the rib by a $16 \mathrm{x}$ microscope objective. The fluorescence emission from the rear end of the waveguide was collected by the same type of objective, passed through 2 OG 550 filters to cut off the residual transmitted pump light and imaged onto a CCD camera linked to a beam-analyzing software. The experimentally observed output profile (Fig. 2) of the fluorescence emission from the rib region demonstrates that a high confinement in both the vertical and horizontal directions was achieved. This profile fits well with the simulated fundamental-mode intensity profile (Fig. 3) at $800 \mathrm{~nm}$ for a $15-\mu \mathrm{m}$ wide by $1.5 \mu \mathrm{m}$ high rib in a $10-\mu \mathrm{m}$ thick Ti:sapphire planar waveguide.

Further optical investigations of our rib structures are under way. These results will be reported at the conference.

\section{References}

[1] A.A. Anderson, R.W. Eason, M. Jelinek, C. Grivas, D. Lane, K. Rogers, L.M.B. Hickey, C. Fotakis, Thin Solid Films 300, 68 (1997).

[2] A.A. Anderson, R.W. Eason, L.M.B. Hickey, M. Jelinek, C. Grivas, D.S. Gill, N.A. Vainos, Opt. Lett. 22, 1556 (1997).

[3] M. Pollnau, R.P. Salathé, T. Bhutta, D.P. Shepherd, R.W. Eason, Opt. Lett. 26, 283 (2001).

[4] J. Lancok, M. Jelinek, J. Bulir, P. Machac, Laser Phys. 8, 303 (1998).

[5] S.H. Park, H. Jeon, Y.J. Sung, G.Y. Yeom, Appl. Opt. 40, 3698 (2001). 